\title{
Design of Novel JNK3 Inhibitors Based on 3D-QSAR In Silico Model
}

\author{
Thirumurthy Madhavan ${ }^{\dagger}$
}

\begin{abstract}
c-Jun N-terminal kinase-3 (JNK-3) has been identified as a promising target for neuronal apoptosis and has the effective therapeutic for neurodegenerative diseases such as Parkinson's disease, Alzheimer's disease, and other CNS disorders. Herein, we report the essential structural and chemical parameters for JNK-3 inhibitors utilizing comparative molecular field similarity indices analysis (CoMSIA) using the derivatives of 3,5-disubstituted quinolines. The best predictions were obtained CoMSIA model ( $\mathrm{q} 2=0.834, \mathrm{r} 2=0.987$ ) and the statistical parameters from the generated 3D-QSAR models were indicated that the data are well fitted and have high predictive ability. The resulting contour map from 3D-QSAR models might be helpful to design novel and more potent JNK3 derivatives.
\end{abstract}

Key words : 3D-QSAR, CoMSIA, JNK3

\section{Introduction}

c-Jun N-terminal Kinase (JNK) are serine threonine protein kinases and members of the mitogen activated protein kinase family (MAPK) $)^{[1-3]}$. JNK is activated by the dual phosphorylation of the motif Thr-Pro-Tyr located in the activation loop. JNK inactivation can be mediated by serine and tyrosine phosphotases ${ }^{[4]}$. JNK activate number of targets including members of the activator protein-1 family (AP-1), JunD, c-Jun, Bcl-2 proteins, c-Myc, activating transcriptional factor 2 (ATF2), and $\mathrm{p} 53^{[5]}$. JNK proteins are encoded by three genes, JNK1, JNK2 and JNK3 and at least 10 different splicing isoforms exist in mammalian cells. JNK1 and JNK2 are widely expressed in tissues whereas JNK3 is selectively expressed in brain, heart and to lesser extent to testis ${ }^{[6]}$. JNK1 plays a key role in linking insulin resistance and obesity and JNK2 plays an important role in autoimmune and inflammatory disease ${ }^{[7]}$. JNK3 has been shown to mediate neuronal apoptosis and make inhibiting this isoform a promising therapeutic target for neurodegenerative diseases such as Parkinson's disease, Alzheimer's disease, and other CNS disorders ${ }^{[8-10]}$. Therefore, identifying JNK3 inhibitor may contribute

Centre for Bioinformatics, Department of Biochemistry, School of life sciences, University of Madras, Guindy campus, Chennai-600025, India

${ }^{\dagger}$ Corresponding author : thiru.murthyunom@gmail.com

(Received: February 5, 2012, Revised : March 25, 2012,

Accepted: March 27, 2012) towards neuroprotection therapies with reduced side effect risks, and will aid in further understanding of the roles of individual JNK kinases. High homology of the ATP-binding site among JNK's makes it challenging to design isoform specific ATP-site directed inhibitors. Therefore, designing selective ATP competitive $\mathrm{JNK}^{[1-3]}$ inhibitors is still a challenging task. Siddiqui and their coworkers have stressed the importance of selectivity for this target ${ }^{[11]}$, our in silico analysis might be the promising step to synthesis highly potent and selective JNK3 analogs. The aim of our study is to optimize the reported derivative set (3,5-disubstituted quinolines) synthesized by Jiang ${ }^{[12]}$ and their coworkers. In this study, we have performed three dimensional quantitative structure activity relationship (3D-QSAR) using CoMSIA $^{[13]}$ techniques for JNK3 inhibitors in order to find the common structural features among them. The present work deals with the ligand-based technique based on the atom-by atom matching to generate reliable 3D-QSAR models. We hope that our theoretical results give some useful reference for the experimentalists in the design of novel and more potent JNK3 inhibitors.

\section{Experimental}

\subsection{Inhibitor Data Set}

The structures of the 4-anilinopyrimidines derivatives and their biological activities of thirty five compounds 
Table 1. Structures and biological activities $\left(\mathrm{pIC}_{50}\right)$ of JNK3 inhibitors

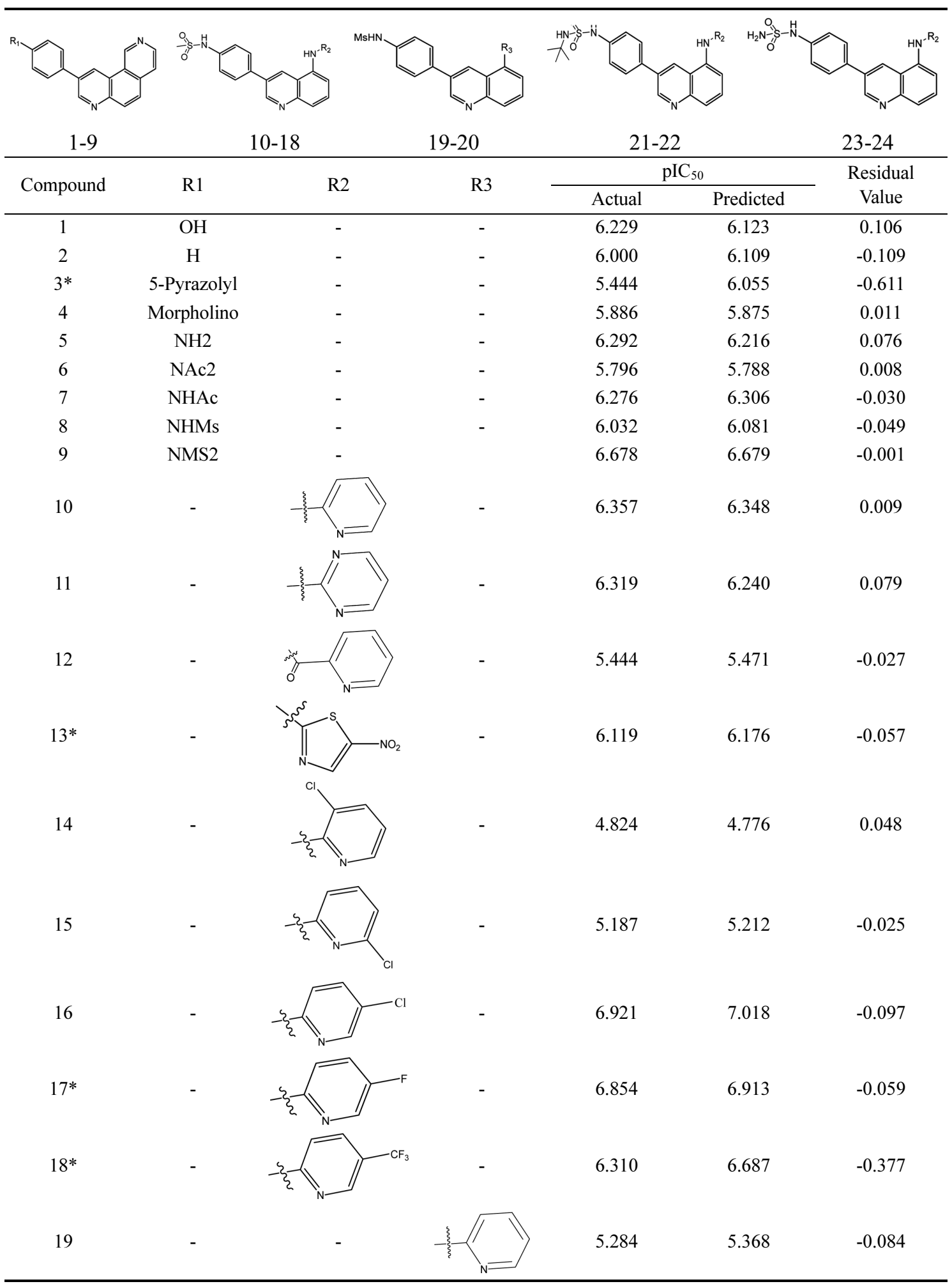


Table 1. Continued

Compound

*Test set compounds

were taken from the literature ${ }^{[12]}$. All original IC50 value of each inhibitor was converted into pIC50 (-logIC50) in order to use the data as dependent variable in CoMSIA model. The test set molecule is truly representative molecule for training set molecules. The test set molecule should cover all the biological activity which is similar to the training set molecule. The total set of compounds was divided into a training set consist of 18 compounds and test set consist of 6 compounds. The selection of training and test sets were done manually so that low, moderate, and high JNK inhibitory activities were all represented. The structures and their activity values are displayed in Table 1.

\subsection{Ligand-based Alignment Method}

In ligand based alignment, the most active molecule was used as template. All rotatable bonds were searched with incremental dihedral angle from $120^{\circ}$ by using systematic search conformation method. Conformational energies were computed with electrostatic term, and the lowest energy conformer was selected as template molecule. Then the template was modified for other ligands of the series. The common moiety was constraint for each molecule and only the varying parts were energy minimized by Tripos force field with Gasteiger-Huckel charge by using conjugate gradient method, and con-

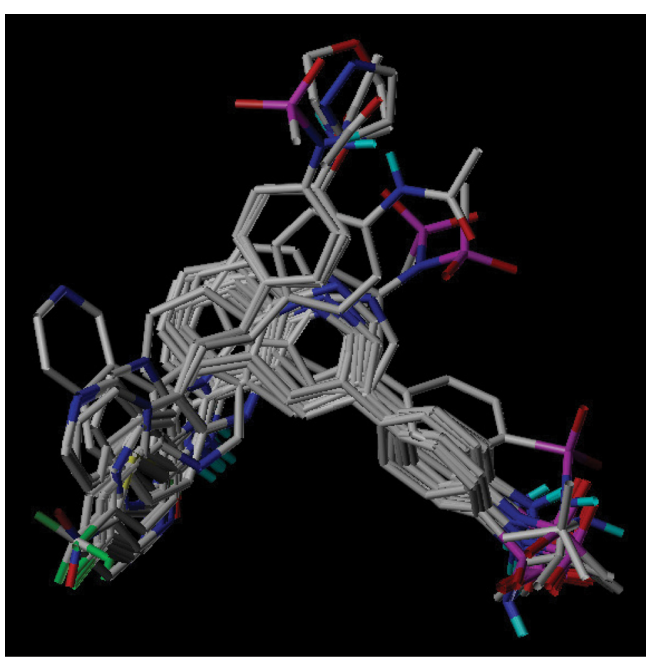

Fig. 1. Alignment of all molecules using atom-by-atom matching. 
vergence criterion was $0.05 \mathrm{kcal} / \mathrm{mol}$ at 10,000 iteration. The minimized structures were aligned over template using atom fit option in Sybyl and subsequently this alignment is used for CoMSIA analysis. The aligned molecules are represented in Fig. 1.

\subsection{Generation of CoMSIA Field}

Sybyl8.1 molecular modeling package is used in this study ${ }^{[14]}$. The molecular alignment was placed in the $3 \mathrm{D}$ grid similar to that of CoMFA analysis. CoMSIA calculated steric and electrostatic fields, in addition to hydrophobic, H-bond donor, and H-bond acceptor fields, and used Gaussian field values. CoMSIA has better ability to visualize and interpret the obtained correlations, in terms of field contributions. A default value of 0.3 was used as attenuation factor

\subsection{Partial Least Square (PLS) Analysis}

The relationship between the structural parameters and the biological activities has been quantified by the PLS algorithm ${ }^{[15,16]}$. CoMSIA descriptors used as independent variables and pIC50 values used as dependent variables in PLS analysis for the generation of 3DQSAR models. To select the best model, the cross-validation procedure was performed using leave one out
(LOO) method, in this procedure one compound was removed from the data set and its activity was predicted using the model build from rest of the data set. It gives cross-validation correlation coefficient (q2) and the optimum number of components. Final analyses i.e. noncross-validation was performed to calculate conventional r2ncv using optimum number of components obtained from cross validation method. The cross-validated coefficient, q2 is calculated using below Equation 1.

$$
q^{2}=1-\frac{\sum\left(Y_{\text {predicted }}-Y_{\text {observed }}\right)^{2}}{\sum\left(Y_{\text {observed }}-Y_{\text {mean }}\right)^{2}}
$$

Ypredicted, Yobserved, and Ymean are predicted, actual, and mean values of the target property (pIC50), respectively. $\Sigma$ (Ypredicted-Yobserved)2 is the predictive sum of squares (PRESS) and the lowest PRESS value is used to derive the final PLS models.

\section{Results and Discussion}

\subsection{CoMSIA Analysis}

A realiable CoMSIA model was derived with the combination of steric, electrostatic and hydrophobic field contribution and Gasteiger-Hückel charge method with $2.0 \AA$ grid space. The Leave one out (LOO) anal-

Table 2. Ligand-based CoMSIA models using different field combination with $2.0 \AA$ grid spacing

\begin{tabular}{|c|c|c|c|c|c|c|c|c|c|c|c|c|}
\hline \multirow[t]{2}{*}{ Model } & \multicolumn{3}{|c|}{$\begin{array}{l}\text { Leave-one-out } \\
\text { cross-validation }\end{array}$} & \multicolumn{3}{|c|}{ Non-cross-validation } & \multirow[t]{2}{*}{$\mathrm{r}_{\text {pred }}^{2}$} & \multicolumn{5}{|c|}{ Field contribution (\%) } \\
\hline & $\mathrm{q}^{2}$ & $\mathrm{n}$ & SDEP & $\mathrm{r}^{2}$ & SEE & F-value & & $\mathrm{S}$ & $\mathrm{E}$ & $\mathrm{H}$ & $\mathrm{D}$ & A \\
\hline SEH & 0.834 & 6 & 0.292 & 0.987 & 0.082 & 137.686 & 0.648 & 0.189 & 0.328 & 0.482 & - & - \\
\hline SEA & 0.673 & 4 & 0.536 & 0.964 & 0.177 & 87.405 & - & 0.140 & 0.420 & - & - & 0.440 \\
\hline SED & 0.713 & 6 & 0.545 & 0.949 & 0.231 & 33.767 & - & 0.300 & 0.615 & - & 0.085 & - \\
\hline EHA & 0.697 & 4 & 0.516 & 0.960 & 0.186 & 78.996 & - & - & 0.307 & 0.298 & - & 0.396 \\
\hline EHD & 0.757 & 6 & 0.502 & 0.962 & 0.198 & 46.679 & - & - & 0.384 & 0.531 & 0.085 & - \\
\hline SHA & 0.629 & 3 & 0.550 & 0.954 & 0.195 & 95.755 & - & 0.165 & - & 0.423 & - & 0.411 \\
\hline SHD & 0.706 & 5 & 0.529 & 0.963 & 0.188 & 61.892 & - & 0.257 & - & 0.633 & 0.110 & - \\
\hline EDA & 0.669 & 5 & 0.561 & 0.955 & 0.208 & 50.510 & - & - & 0.435 & - & 0.089 & 0.476 \\
\hline SDA & 0.477 & 6 & 0.737 & 0.947 & 0.234 & 33.001 & - & 0.283 & - & - & 0.115 & 0.602 \\
\hline SEHD & 0.765 & 6 & 0.493 & 0.973 & 0.168 & 65.954 & - & 0.169 & 0.329 & 0.442 & 0.059 & - \\
\hline SEHA & 0.702 & 3 & 0.493 & 0.951 & 0.201 & 89.693 & - & 0.109 & 0.285 & 0.285 & - & 0.342 \\
\hline SEDA & 0.693 & 5 & 0.540 & 0.956 & 0.204 & 52.447 & - & 0.123 & 0.387 & - & 0.078 & 0.413 \\
\hline EHAD & 0.699 & 5 & 0.535 & 0.969 & 0.172 & 75.059 & - & - & 0.280 & 0.280 & 0.093 & 0.347 \\
\hline SEHAD & 0.697 & 6 & 0.560 & 0.981 & 0.141 & 93.960 & - & 0.091 & 0.259 & 0.264 & 0.077 & 0.309 \\
\hline
\end{tabular}

S, steric field, E, electrostatic field, H, hydrophobic field, D, hydrogen bond donor, A, hydrogen bond acceptor; n, number of statistical components; $\mathrm{q}^{2}$, crossvalidated correlation coefficient; $\mathrm{r}^{2}$, non-cross-validated correlation coefficient; SEE, standard estimated error; F, Fisher value; $\mathrm{r}^{2}$ pred = predictive correlation coefficient for test set; shaded box indicated that the model utilized for 3D-QSAR parameters. 


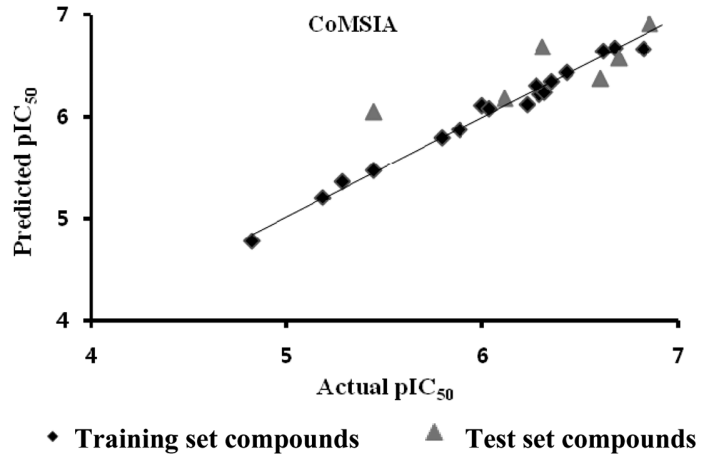

Fig. 2. Plot of actual versus predicted pIC50 values of the training set and test set compounds.

ysis gave the cross-validated q2 of 0.834 with 6 components and noncross-validated PLS analysis resulted in a correlation coefficient $\mathrm{r} 2$ of $0.987, \mathrm{~F}=137.686$, and an estimated standard error of 0.082 . Statistical results obtained from the constructed model verified the predictive ability of the model (Table 2) and further implied that the steric, electrostatic, and hydrophobic factors contribute to the binding affinities. The predictive ability of the developed CoMSIA model was assessed by the test set (six molecules) predictions, which were excluded during CoMSIA model generation. The predictive ability of the test set was 0.648 . Predicted and experimental activities and their residual values of all inhibitors are shown in Table 1, and the corresponding scatter plot is depicted in Fig. 2.

\subsection{CoMSIA Contour Map}

The CoMSIA contour map was generated based on the ligand-based (atom-by atom matching) alignment method. The CoMSIA result is usually represented as 3D 'coefficient contour' map. It shows regions where variations of steric, electrostatic and hydrophobic nature in the structural features of the different molecules contained in the training set lead to increase or decrease in the activity.

The steric interaction is represented by green and yellow contours, in which green colored regions indicate areas where increased steric bulk is associated with increased activity, and yellow regions suggested that where steric bulk is unfavorable to activity. The steric contour map is displayed in Fig. 3.

The green steric contour near the R2 position of the phenyl ring indicates that substitution of bulky group is

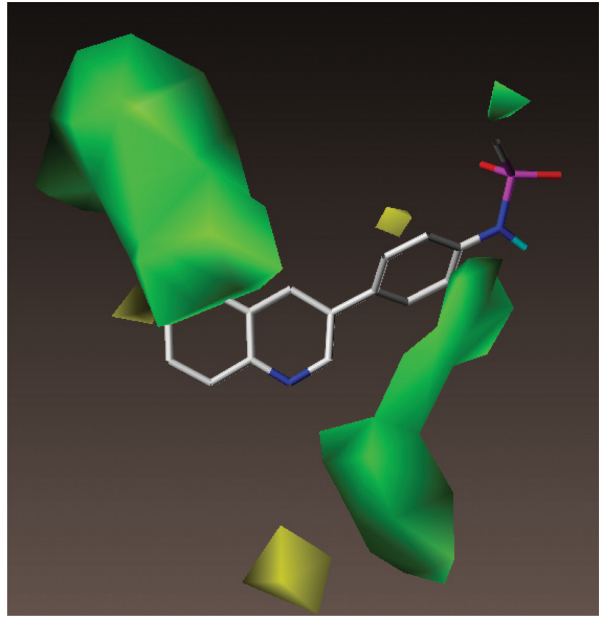

Fig. 3. CoMSIA contour maps for steric field with highly active compound 16, where green contour indicates regions where bulky groups increases activity and yellow contours indicates bulky groups decreases activity.

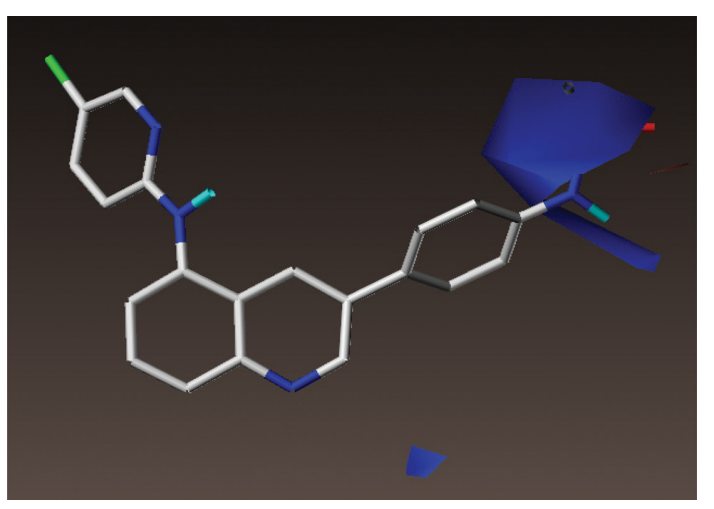

Fig. 4. CoMSIA contour maps for electrostatic field with highly active compound 16 , where blue contour indicates regions where electropositive groups increases activity and red contours indicates regions where electronegative groups increases activity.

preferred at this position. This may be the reason that compounds $10-18$ with bulkier substituent at this position are more active. There was a yellow contour region which was very close to the green contour map in R1 position; the contour map clearly indicated that substitution of bulkier groups would decrease the activity. This may be the reason that compounds 3 , and 4 having bulkier substitution shows less activity. The electrostatic interaction is represented by red and blue contours (Fig. 4), among which blue colored regions show areas where 


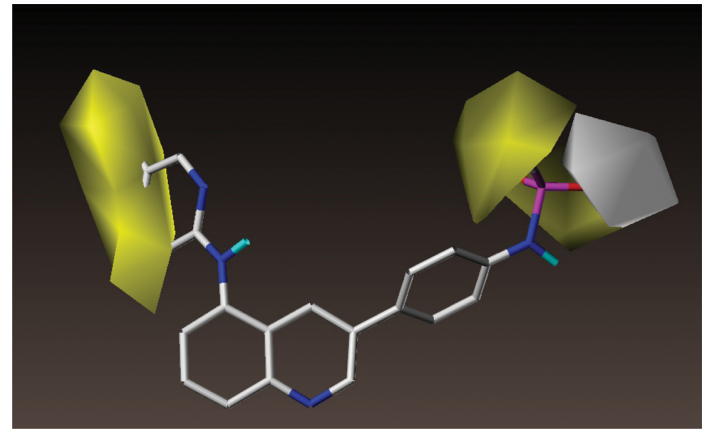

Fig. 5. CoMSIA hydrophobic contour map with compound 16. Yellow contours indicate the regions where hydrophobic groups increase activity, whereas white contours indicate the regions where hydrophobic groups decrease activity.

more positively charged groups are favored, and red region highlight areas where groups with more negative charges are favored. These contour maps give us some general insight into the nature of the receptor-ligand binding region.

The electrostatic contour plot shows that there is a blue colored region situated close to the $\mathrm{R} 2$ positions. Substitution of electron donating group in para position of R2 phenyl group increases the biological activity. It indicates that the electropositive charges in these regions are very important for ligand binding, and electropositive group linked to this position will enhance the biological activity. The hydrophobhic contour map is displayed in Fig. 5.

The yellow and white contour map indicates the regions where hydrophobic and hydrophilic groups are preferred, respectively. There were two yellow contours observed near to the R2 position of the phenyl ring. This explains why the substitution of the hydrophobic groups could improve the potency of compounds. These hydrophobic contour maps are correlating well with the steric contour map, and these may the reason that compounds 10-18 shows higher biological activity.

\section{Conclusion}

In this work, we have developed a satisfactory 3DQSAR models from 3,5-disubstituted quinolines derivatives using CoMSIA method based on atom-by-atom matching alignment. The contour map indicated important sites, such as steric, electrostatic and hydrophobic factors can significantly affect the bioactivities of the compounds. The steric and hydrophobic contour map indicated that substitution of bulkier groups in the R2 position of the phenyl ring would enhance the biological activity. In addition to this, the electrostatic contour map shows that the substitution of electron donating group in R2 position could improve the biological activity. Our 3D-QSAR model give the robust information to understand the structural and chemical features which are necessary for designing and finding new potential JNK3 inhibitors.

\section{References}

[1] J. M. Kyriakis and J. Avruch, "Pp54 microtubuleassociated protein 2 kinase", J. Biol. Chem., Vol. 265, pp. 17355-17363, 1990.

[2] M. Hibi, A. Lin, T. Smeal, A. Minden, and M. Karin, "Identification of an oncoprotein and UVresponsive protein kinase that binds and potentiates the c-Jun activation domain", Genes and Development., Vol. 7, pp. 2135-2148, 1993.

[3] V. Adler, A. Polotskaya, F. Wagner, and A. S. Kraft, "Affinity-purified c-Jun amino-terminal protein kinase requires serine/threonine phosphorylation for activity”, J. Biol. Chem., Vol. 267, pp. 17001-17005, 1992.

[4] R. J. Davis, "Signal transduction by the JNK group of MAP kinases", Cell., Vol. 103, pp. 239-252, 2000.

[5] A. M. Bode and Z. Dong, "The functional contrariety of JNK", Molecular carcinogenesis., Vol. 46, pp. 591-598, 2007.

[6] S. Gupta, T. Barrett, A. J. Whitmarsh, J. Cavanagh, H.K. Sluss, B. Derijard, and R. J. Davis, "Selective interaction of JNK protein kinase isoforms with transcription factors", EMBO J., Vol. 15, No. 11, pp. 2760-2770.

[7] J. H. Martin, A. A. Mohit, and C. A. Miller, "Developmental expression in the mouse nervous system of the p493F12 SAP kinase", Mol. Brain Res., Vol. 35, pp. 47-57, 1996.

[8] C. Dong, D. D. Yang, M. Wysk, A. J. Whitmarsh, R. J. Davis, and R. A. Flavell, "Defective T cell differentiation in the absence of Jnk1", Science., Vol. 282, pp. 2092-2095, 1998.

[9] J. M. Kyriakis and J. Avruch, "Mammalian mitogen-activated protein kinase signal transduction pathways activated by stress and inflammation", Physiol. Rev., Vol. 81, pp. 807-869, 2001.

[10] G.Y. Zhang, and Q. G. Zhang, "Agents targeting c- 
Jun N-terminal kinase pathway as potential neuroprotectants", Expert Opin. Invest. Drugs., Vol. 14, pp. 1373-1383, 2005.

[11] J. M. A. Siddiqui and P. A. Reddy, "Small Molecule JNK (c-Jun N-Terminal Kinase) Inhibitors", J. Med. Chem., Vol. 53, pp. 3005-3012, 2010.

[12] R. Jiang, D. Duckett, W. Chen, J. Habel, Y. Y. Ling, P. L. Grassob, and T. M. Kamenecka, "3,5-Disubstituted quinolines as novel c-Jun N-terminal kinase inhibitors", Bioorganic and Medicinal Chemistry Letters., Vol. 17, pp. 6378-6382, 2007.

[13] G. Klebe, U. Abraham, and T. Mietzner, "Molecular similarity indices in a comparative analysis (CoM-
SIA) of drug molecules to correlate and predict their biological activity", J. Med. Chem., Vol. 37, pp. 4130-4146, 1994.

[14] Sybyl 8.1, Tripos Inc., St. Louis, MO 63144, USA.

[15] S. J. Cho and A. Tropsha, "Cross-validated R2guided region selection for comparative molecular field analysis: A simple method to achieve consistent results", J. Med. Chem., Vol. 38, pp.1060-1066, 1995.

[16] S. Wold, M. Sjostrom, and L. Eriksson, "PLSregression: a basic tool of chemometrics", Chemometrics and intell. lab. Sy., Vol. 58, pp. 109-130, 2001. 